\title{
The clinical data from 19 critically ill patients with coronavirus disease 2019: a single-centered, retrospective, observational study
}

\author{
Jinping Zhang ${ }^{1} \cdot$ Peng Liu ${ }^{2} \cdot$ Morong Wang ${ }^{1} \cdot$ Jie Wang ${ }^{1} \cdot$ Jie Chen ${ }^{1} \cdot$ Wenling Yuan ${ }^{1} \cdot$ Mei Li $^{1} \cdot$ Zhijuan Xie $^{1}$. \\ Wangping Dong ${ }^{1} \cdot$ Hongye $\mathrm{Li}^{1} \cdot$ Yan Zhao ${ }^{1} \cdot$ Lun Wan $^{1} \cdot$ Tian Chu $^{1} \cdot$ Lu Wang $^{1} \cdot$ Hui Zhang ${ }^{1} \cdot$ Ting Tao $^{1} \cdot$ Jing Ma $^{1}$
}

Received: 15 March 2020 / Accepted: 2 April 2020 / Published online: 21 April 2020

(C) Springer-Verlag GmbH Germany, part of Springer Nature 2020

\begin{abstract}
Background The objectives of this study were to analyze the clinical features of coronavirus disease 2019 (COVID-19) and evaluate the diagnosis and treatment.

Methods A retrospective analysis of the clinical manifestation and auxiliary examination of 19 patients with COVID-19 from the Liyuan Hospital intensive care unit (ICU) between January 16, 2020 and February 20, 2020 was undertaken.

Results There were 11 male and 8 female cases among the patients. The median (range) age was 73 (38-91) years. Of these patients, 8 (42.1\%) had died and the median duration from ICU admission to death was 2 (interquartile range (IQR): 1-10.75) days. Seven of these 8 patients had underlying diseases. The auxiliary examination showed fever (68.4\%), dry cough (15.8\%), dyspnea (10.5\%), and diarrhea (5.3\%). All 19 cases showed ground-glass changes on chest computed tomography. Serum hypersensitive C-reactive protein (hs-CRP) and serum amylase A (SAA) were clearly increased in all of the cases. Among the 19 cases, there were $16(84.2 \%)$ cases in which the total number of lymphocytes decreased, 12 cases $(63 \%)$ had reduced liver function, and 11 cases (58\%) had deviant results for fibrinogen (FIB) and D-dimer, in particular, the D-dimer level was significantly higher in the non-survivors compared with the survivors.

Conclusion There were more men than women among critically ill patients. All of the cases showed ground-glass changes on chest computed tomography and the vast majority of patients displayed fever and dry cough. The clinical laboratory indices change significantly, especially the D-dimer level among non-survivors.
\end{abstract}

Keywords Coronavirus disease $2019 \cdot$ Clinical manifestations $\cdot$ Epidemiology $\cdot$ Retrospective $\cdot$ D-dimer

Jinping Zhang and Peng Liu contributed equally to this work.

Jinping Zhang

zhangjinping0425@163.com

Jing Ma

jiaositang@163.com

1 ICU, Liyuan Hospital affiliated to Tongji Medical College, Huazhong University of Science and Technology, Wuhan 430071, China

2 State Key Laboratory of Virology and Hubei Province Key Laboratory of Allergy and Immune-related Diseases, Department of Immunology, Wuhan University School of Basic Medical Sciences, Wuhan 430071, China

\section{Introduction}

Since December 2019, an outbreak of unexplained pneumonia in Wuhan, China, has spread rapidly to other provinces in China and around the world (Chen et al. 2020; Holshue et al. 2020). Studies have indicated that it is a novel coronavirus pneumonia, which was named coronavirus disease 2019 (COVID-19). As of February 24, 2020, the total number of patients has risen sharply to 77,262 in China, with 2595 (3.2\%) who have died. The main diagnosis method is the pathogenic results obtained by quantitative real-time polymerase chain reaction (qRT-PCR), which proved to be highly homologous with known genetic sequencing. However, the positivity rate is $30-50 \%$, due to the influence of individual factors, the onset time, and specimen collection. It is very important to discover the specific information characterizing critically ill patients (Chung et al. 2020; Xie et al. 2020). 
Therefore, we conducted a retrospective analysis of critically ill patients with COVID-19.

\section{Methods}

All of the COVID-19 cases in this study came from the Liyuan Hospital intensive care unit (ICU) of Tongji Medical College, Huazhong University of Science and Technology, during the period January 16, 2020 to February 20, 2020.

According to the World Health Organization's (WHO) interim guidance, a severely ill patient is defined as one with any of the following symptoms:

1. Respiratory distress, respiratory rate (RR) acuity of 30 breaths/min.

2. Oxygen saturation during resting state of $93 \%$ or less.

3. Arterial blood oxygen partial pressure $\left(\mathrm{PaO}_{2}\right) /\left(\mathrm{FiO}_{2}\right)$ $\leq 300 \mathrm{mmHg}$ (high-altitude region (more than $1000 \mathrm{~m}$ above sea level, according to the following formula for correction: $\mathrm{PaO}_{2} / \mathrm{FiO}_{2} \times 760$ (mmHg)/atmosphere)).

Critically ill patients are defined as those with any of the characteristics:

1. Respiratory failure and need for mechanical ventilation.

2. Shock.

3. Combination of failure of other organ functions, followed by the need for ICU admission.

We collected data on age, sex, underlying diseases (chronic pulmonary disease, chronic cardiac disease, cerebrovascular disease, diabetes, etc), symptoms, vital signs (heart rate, blood pressure, respiratory rate, etc.), and laboratory values (blood, routine urine test, liver function, renal function, electrolyte and myocardial enzymes, troponin, coagulation function, hypersensitive C-reactive protein (hs-CRP), and serum amylase A (SAA)).

\section{Results}

Among the COVID-19 patients, there were 11 males and 8 females. Their median (range) age was 73 (38-91) years. Of these patients, $8(42.1 \%)$ died and the median duration from ICU admission to death was 2 (interquartile range (IQR): 110.75 ) days. Fifteen $(78.9 \%)$ patients had underlying diseases, mainly high blood pressure or diabetes. Seven of the patients who died had underlying diseases. All of the patients were citizens of Wuhan, China, and had no history of contact with COVID-19 (Table 1).

Clinical manifestations included fever $(13 / 19,68.4 \%)$, dry cough $(3 / 19,15.8 \%)$, dyspnea $(2 / 19,10.5 \%)$, and diarrhea
$(1 / 19,5.3 \%)$. Seven patients were treated by intubation and mechanical ventilation.

Auxiliary examination showed that all 19 cases (100\%) showed ground-glass changes on chest computed tomography (CT). The clinical laboratory indices were as follows: the infection indices (hs-CRP and SAA) were clearly increased in all 19 cases; there were 16 cases $(84.2 \%)$ in which the total number of lymphocytes decreased; 12 cases $(63 \%)$ had decreased liver function; 11 cases (58\%) had deviant results for fibrinogen (FIB) and D-dimer, in particular, the increase in the D-dimer level was significantly higher among the nonsurvivors $(n=8,100 \%)$ compared to the survivors $(n=11$, 27\%); 9 cases (47\%) had deviant levels of cardiac muscle enzymes and troponin $\mathrm{T}$ (Table 2).

\section{Discussion}

At present, there are no specific drugs for COVID-19, only general supportive therapy and treatment in isolation. For mildly ill patients, the infection usually clears spontaneously. Nevertheless, severe illnesses develop easily, such as acute respiratory distress syndrome (ARDS), multiple system organ failure, or even death. In this study, more men than women and more older than younger individuals made up the group of critically ill patients, and the median (range) age of the 19 patients was 73 (38-91) years. Eight (42.1\%) patients died and the median duration from ICU admission to death was 2 (IQR: 1-10.75) days. These results suggest that sex and age are affecting the risk factors. Patients with a history of underlying disease (high blood pressure or diabetes) are at increased risk of becoming critically ill or dying if they have the COVID-19 infection. These findings are consistent with previous reports (Chen et al. 2020; Huang et al. 2020). The susceptibility of a population to infection by this new virus drives the dynamics of a pandemic. The clinical manifestations include fever, fatigue, and dry cough without phlegm. A few patients have nasal congestion, runny nose, sore throat, and diarrhea (Cui et al.2019; Zhu et al. 2020). Critically ill patients were more vulnerable to dyspnea, hypoxemia, and even ARDS, irreformable metabolic acidosis, sepsis, and coagulation disturbance after just one week (Wang et al. 2020). Our study found that the severely ill patients went to the hospital early, at the first signs of symptoms of fever and cough, and the time from showing symptoms to ICU admission was 6.4 days. At the ICU, 18 cases (94.7\%) developed ARDS rapidly.

In regards to the laboratory tests, all 19 cases $(100 \%)$ showed ground-glass changes on chest $\mathrm{CT}$. The vast majority of these clinical laboratory indices changed significantly: the hs-CRP and SAA were clearly increased in all 19 cases (100\%); 9 cases (47\%) had deviant levels of cardiac muscle enzymes and troponin T; 12 cases $(63 \%)$ had decreased liver function; 11 cases (58\%) had deviant results for FIB and D- 
Table 1 Characteristics of the study population

\begin{tabular}{llll}
\hline Groups & \multicolumn{2}{l}{ Critically ill patients $(n=19)$} & \\
\cline { 2 - 4 } & Survivors $(n=11)$ & Non-survivors $(n=8)$ & All patients $(n=19)$ \\
\hline Age, median (range) & $68(38-87)$ & $77(66-91)$ & $73(38-91)$ \\
Males/females & $6 / 5$ & $5 / 3$ & $11 / 8$ \\
Clinical symptoms & $11 / 11$ & $8 / 8$ & $19 / 19$ \\
Chest CT (positive/tested) & $11 / 11$ & $8 / 8$ & $19 / 19$ \\
qRT-PCR assay (positive/tested) & $10 / 11$ & $3 / 8$ & $13 / 19$ \\
Hypertension & $6 / 11$ & $5 / 8$ & $11 / 19$ \\
Diabetes & $1 / 11$ & $3 / 8$ & $4 / 19$ \\
Chronic bronchitis & $3 / 11$ & $0 / 8$ & $3 / 19$ \\
Chronic cardiac disease & $0 / 11$ & $3 / 8$ & $3 / 19$ \\
Cerebrovascular disease & $1 / 11$ & $2 / 8$ & $3 / 19$ \\
\hline
\end{tabular}

COVID-19 clinical symptoms include fever, cough, and lacking in strength dimer, in particular, the increase in the D-dimer level was significantly higher among the non-survivors $(n=8,100 \%)$ compared to the survivors $(n=11,27 \%)$. This suggested that the D-dimer level can be used to monitor change in the condition of severely ill patients (Fig. 1). There were 16 (84.2\%) cases in which the total number of lymphocytes decreased, which may prompt the theory that COVID-19 infects cells of the human immune system, destroying or impairing their function.

Previous studies showed that the coronavirus can induce the body to produce oxidative stress and release a large amount of active oxygen free radicals, which can, on one hand, make the virus replicate unceasingly, but, on the other hand, the excessive free radicals can damage the body's biological membrane lipid peroxidation, enzyme, amino acid, and oxidative protein, leading to injury to the organs, such as the lung, heart, liver, kidney, etc. (Li et al 2016; Honce and Schultz-Cherry 2019; Li et al. 2020; Zhu et al. 2019).

COVID-19 belongs to the group of viruses which can easily mutate during the process of RNA replication. As a result, we suspect that the new coronavirus in patients with multiple organ function damage is most likely related to this group. In regards to imaging aspects, 19 cases $(100 \%)$ showed groundglass changes on chest $\mathrm{CT}$, which is the best tool to use to evaluate the imaging signs for early diagnosis.

In conclusion, to analyze the clinical features of patients with COVID-19, we found that sex and age are affecting the risk factors. Patients with a history of underlying disease (high blood pressure or diabetes) are at increased risk of becoming critically ill or dying if they have the COVID-19 infection. Chest CT, lymphocyte count, hs-CRP, and SAA can be used in disease diagnosis. The D-dimer level plays an important

Table 2 Symptoms and clinical laboratory index results of critically ill patients with COVID-19 pneumonia

\begin{tabular}{|c|c|c|c|}
\hline \multirow[t]{2}{*}{ Groups } & \multicolumn{3}{|c|}{ Critically ill patients $(n=19)$} \\
\hline & Survivors $(n=11)$ & Non-survivors $(n=8)$ & All patients $(n=19)$ \\
\hline \multicolumn{4}{|l|}{ Symptoms } \\
\hline Fever & $7 / 11$ & $6 / 8$ & $13 / 19$ \\
\hline Cough & $5 / 11$ & $3 / 8$ & $8 / 19$ \\
\hline Lacking in strength & $2 / 11$ & $0 / 8$ & $2 / 19$ \\
\hline Dyspnea & $0 / 11$ & $2 / 8$ & $2 / 19$ \\
\hline \multicolumn{4}{|l|}{ Clinical laboratory index, median (IQR) } \\
\hline Hypersensitive C-reactive protein $(\mathrm{mg} / \mathrm{L})$ & $86.9(47.9-120.5)$ & $166.15(82.3-129.4)$ & $95.4(54.1-120.5)$ \\
\hline Serum amylase A (mg/L) & $65.7(56.9-100.8)$ & $95.1(54.8-79.8)$ & $64.3(56.4-94.6)$ \\
\hline Aminotransferase (U/L) & $39.5(26.4-77.5)$ & $50.9(43.2-71.2)$ & $47.2(29.7-75.8)$ \\
\hline Albumin $(\mathrm{g} / \mathrm{L})$ & $36(31.8-37.3)$ & $33.65(29.2-34.7)$ & $34.1(30.9-36.7)$ \\
\hline D-dimer (mg/L FEU) & $0.48(0.42-0.97)$ & $2.15(1.4-9.2)$ & $0.91(0.475-2.35)$ \\
\hline
\end{tabular}

COVID-19 clinical symptoms include fever, cough, and lacking in strength 


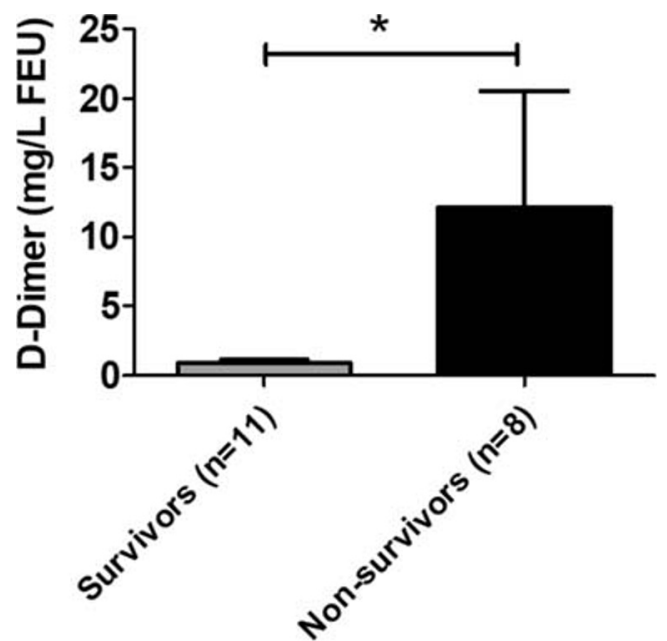

Fig. 1 Comparison of the D-dimer levels among non-survivors and survivors. $* p<0.05$ (Mann-Whitney $U$-test)

role in critically ill patients and it is suggested that D-dimer can be used to monitor change in the condition of severely ill patients. There are certain limitations to this study, in that the number of patients was small and it should be classified as a retrospective study. We hope to have more samples in future to carry out a more comprehensive study and elucidate better means for the clinical treatment of COVID-19.

Acknowledgements We thank the medical staff of Liyuan Hospital for their assistance in collecting the clinical samples.

\section{Compliance with ethical standards}

Conflict of interest The authors declare that they have no conflicts of interest related to this work. We declare that we do not have any commercial or associative interest that represents a conflict of interest in connection with the work submitted.

\section{References}

Chen N, Zhou M, Dong X et al (2020) Epidemiological and clinical characteristics of 99 cases of 2019 novel coronavirus pneumonia in Wuhan, China: a descriptive study. Lancet 395(10223):507-513

Chung M, Bernheim A, Mei X et al (2020) CT imaging features of 2019 novel coronavirus (2019-nCoV). Radiology 295:202-207. https:// doi.org/10.1148/radiol.2020200230

Cui J, Li F, Shi ZL (2019) Origin and evolution of pathogenic coronaviruses. Nat Rev Microbiol 17(3):181-192

Holshue ML, DeBolt C, Lindquist S et al (2020) First case of 2019 novel coronavirus in the United States. N Engl J Med 382(10):929-936

Honce R, Schultz-Cherry S (2019) Impact of obesity on influenza a virus pathogenesis, immune response, and evolution. Front Immunol 10: 1071

Huang C, Wang Y, Li X et al (2020) Clinical features of patients infected with 2019 novel coronavirus in Wuhan, China. Lancet 395(10223): 497-506

Li S, Li H, Xu X, Saw PE, Zhang L (2020) Nanocarrier-mediated antioxidant delivery for liver diseases. Theranostics 10(3):1262-1280

Li SW, Wang CY, Jou YJ et al (2016) SARS coronavirus papain-like protease induces Egr-1-dependent up-regulation of TGF- $\beta 1$ via ROS/p38 MAPK/STAT3 pathway. Sci Rep 6:25754

Wang D, Hu B, Hu C et al (2020) Clinical characteristics of 138 hospitalized patients with 2019 novel coronavirus-infected pneumonia in Wuhan, China. JAMA (in press). https://doi.org/10.1001/jama. 2020.1585

Xie X, Zhong Z, Zhao W et al (2020) Chest CT for typical 2019-nCoV pneumonia: relationship to negative RT-PCR testing. Radiology. https://doi.org/10.1148/radiol.2020200343

Zhu N, Zhang D, Wang W et al (2020) A novel coronavirus from patients with pneumonia in China, 2019. N Engl J Med 382(8):727-733

Zhu Y, Wei J, Liu Y et al (2019) Assessing the effect on the generation of environmentally persistent free radicals in hydrothermal carbonization of sewage. Sci Rep 9(1):17092

Publisher's note Springer Nature remains neutral with regard to jurisdictional claims in published maps and institutional affiliations. 